\title{
Strategies for Optimization of Cryogenic Electron Tomography Data Acquisition
}

\author{
Felix Weis ${ }^{1}$, Wim J. H. Hagen ${ }^{1}$, Martin Schorb ${ }^{2}$, Simone Mattei ${ }^{1,3}$ \\ ${ }^{1}$ Structural and Computational Biology Unit, European Molecular Biology Laboratory ${ }^{2}$ Electron Microscopy Core Facility, European Molecular Biology \\ Laboratory ${ }^{3}$ Imaging Centre, European Molecular Biology Laboratory
}

\section{Corresponding Author}

Simone Mattei

simone.mattei@embl.de

\section{Citation}

Weis, F., Hagen, W.J.H., Schorb, M., Mattei, S. Strategies for Optimization of Cryogenic Electron Tomography Data Acquisition. J. Vis. Exp. (169), e62383, doi:10.3791/62383 (2021).

\section{Date Published}

March 19, 2021

\section{DOI}

$10.3791 / 62383$

\section{URL}

jove.com/video/62383

\section{Abstract}

Cryogenic electron tomography (cryoET) is a powerful method to study the $3 \mathrm{D}$ structure of biological samples in a close-to-native state. Current state-of-the-art cryoET combined with subtomogram averaging analysis enables the high-resolution structural determination of macromolecular complexes that are present in multiple copies within tomographic reconstructions. Tomographic experiments usually require a vast amount of tilt series to be acquired by means of high-end transmission electron microscopes with important operational running-costs. Although the throughput and reliability of automated data acquisition routines have constantly improved over the recent years, the process of selecting regions of interest at which a tilt series will be acquired cannot be easily automated and it still relies on the user's manual input. Therefore, the set-up of a large-scale data collection session is a timeconsuming procedure that can considerably reduce the remaining microscope time available for tilt series acquisition. Here, the protocol describes the recently developed implementations based on the SerialEM package and the PyEM software that significantly improve the time-efficiency of grid screening and large-scale tilt series data collection. The presented protocol illustrates how to use SerialEM scripting functionalities to fully automate grid mapping, grid square mapping, and tilt series acquisition. Furthermore, the protocol describes how to use PyEM to select additional acquisition targets in off-line mode after automated data collection is initiated. To illustrate this protocol, its application in the context of high-end data collection of Sars-Cov-2 tilt series is described. The presented pipeline is particularly suited to maximizing the time-efficiency of tomography experiments that require a careful selection of acquisition targets and at the same time a large amount of tilt series to be collected. 
Cryogenic electron microscopy (cryoEM) methods are based on the imaging of biological samples by means of a transmission electron microscope (TEM) after their rapid vitrification, a sample preparation process that preserves the molecular and cellular structures of specimens in a close-to-native and hydrated state ${ }^{1,2}$. In cryogenic electron tomography (cryoET) a 3D model of the vitrified sample is achieved by acquiring a number of images of the same region of interest from different orientations, the so-called tilt series, followed by the computational reconstruction of the tomographic volume ${ }^{3}$. This advanced imaging technique has matured into a powerful method for the structural investigation of biological processes in the context of their native cellular environments ${ }^{4,5,6}$.

In addition to the ultrastructural analysis of the vitrified sample, high-resolution reconstructions of macromolecular complexes that are present in multiple copies within the tomographic volume can be obtained by applying subtomogram averaging ${ }^{5}$. This reconstruction approach is based on the iterative alignment and averaging of subvolumes containing the structure of interest and it is aimed at increasing the signal-to-noise ratio and the resolution of the final reconstruction ${ }^{7,8}$. Subtomogram averaging relies on the collection and processing of a large amount of data that often demands the acquisition of hundreds of tilt series by means of high-end TEMs with onerous operational running-costs.

Currently the setup of such automated cryoET sessions is a time-consuming process that usually relies on the user's manual input ${ }^{9,10,11}$. Typically, targets are identified by visual inspection of the mapped grid and subsequently set up for automated data collection. The user's efficiency in identifying acquisition points is often affected by the nature of the sample, becoming particularly challenging when analyzing purified macromolecules with suboptimal concentration or in the case of rare events within crowded cellular environments, implying the use of correlative approaches ${ }^{12}$. Furthermore, current workflows require the acquisition of images during set-up at various magnifications that will be later used for precise localization and centering of the target during automated acquisition ${ }^{11,13,14}$. These high-precision re-alignment steps are crucial for high-resolution applications, which demand the imaging to be performed at high magnification and require accurate centering steps for retaining the region of interest within the resulting small field of view. Altogether, several hours of each data collection session are committed for this time-consuming procedure during which the TEM is not engaged in tilt series acquisition. Therefore, depending on the amount of tilt series required, the identification and set-up of acquisition points can have a considerable impact on the microscope time available for data collection during a cryoET session.

Described here is an optimized protocol based on the SerialEM software package ${ }^{15}$ and the latest version the PyEM software ${ }^{16}$ to map grids, map grid squares, select targets, and set up automated data acquisition for large-scale tilt series collection. The key concept of this approach is to provide SerialEM with computationally generated images by PyEM for each acquisition item, termed virtual maps, for the accurate localization and centering of the target. To gain actual acquisition time, the selection of the targets as well as the creation of the virtual maps are performed off-line using a second dummy instance of SerialEM, decoupling the selection process of acquisition targets from TEM operations. 
While not addressing how to increase the data quality ${ }^{13,17}$ or the speed of tilt series acquisition ${ }^{18,19}$, this protocol is primarily focused on strategies to optimize the timeefficiency of large-scale automated cryoET sessions setup. Therefore, the implementation of the presented protocol is meant for those scientists establishing cryoET data collection workflows that desire to maximize the yield of automated data acquisition by increasing the microscope time available for tilt series acquisition.

\section{Protocol}

The protocol described here is part of a more comprehensive document produced by the EMBL CryoEM Service Platform that includes thorough step-by-step instructions and screenshots illustrating the entire procedure of a typical cryoET session, including sample loading, grid mapping, microscope tuning, set up of acquisition points, and automated data collection. The full protocol can be downloaded at the following link: https://oc.embl.de/ index.php/s/9OuTI8AazDkCNq0/download

\section{Prerequisites}

1. Install SerialEM version 3.8 and set up to control the microscope and detector (http://bio3d.colorado.edu/ SerialEM/betaHlp/html/setting_up_serialem.htm).

2. Install a dummy instance of SerialEMversion 3.8 (https://sphinx-emdocs.readthedocs.io/en/latest/ serialEM-note-setup-dummy.html).

3. Install PyEM (https://git.embl.de/schorb/pyem).

\section{Grid mapping}

1. Load a cassette with grids into the microscope autoloader.
2. Set up imaging conditions suitable for mapping the entire grid. Do this at the lowest magnification possible taking into account the field of view on the detector used (EFTEM SA 2250x). Save the imaging conditions as a SerialEM Imaging State to make things convenient for later use.

3. Set up full montage

1. Open the SerialEM Navigator menu.

\section{Select Montaging \& Grids.}

\section{Select Set up Full Montage.}

4. Start the script Grid_Mapping. Allow the script to wait for the autoloader to cool down; do an inventory and then map each grid that the inventory procedure found. Input an email via the Tilt Series Menu to allow the script to conveniently send an email when done.

5. Save Navigator.

6. Inspect all grid maps from the SerialEM Navigator and choose which grid to map further at higher magnification. NOTE: Many TEMs equipped with an autoloader system will show a rotation of the grid when reloaded. It's best to remap any grid after reloading it. Alternative systems usually only suffer some shifts, which can be corrected in SerialEM with a Shift To Marker procedure.

\section{Set up the SerialEM low dose}

1. Set the magnification of SerialEM low dose View and Preview modes (this is required for step 6).

2. Acquire a View image, and save it as a map in the Navigator.

3. Acquire a Preview image, and save it as a map in the Navigator. 
1. Set both the modes to the same binning (binning 4 is suggested). This allows saving the two maps into one image stack.

2. In the SerialEM Navigator window, change the label of the View map to View and the label of the Preview map to Preview.

3. Save and close the navigator file.

NOTE: No target is needed for the initial View and Preview images, PyEM will merely use their imaging settings.

\section{Grid square mapping}

1. Set up the imaging conditions to map grid squares. It is of great importance to be able to see the sample of interest and the fiducial bead distribution. To ensure optimal contrast for grid square maps, perform the following steps.

1. Insert an objective aperture.

2. If applicable, insert the energy filter slit.

3. Use a defocus of $-100 \mu \mathrm{m}$.

2. Screen for good grid squares suitable for further mapping. After visual inspection of the squares from the grid map, take test images of the square with the imaging conditions to be used for grid square maps.

3. When a good square is identified, mark its center in the grid map image using the Add Points feature of the SerialEM Navigator.

4. Once all the points are added, in the SerialEM Navigator press Shift + A on the first point, then press Shift + A on the last point.

NOTE: All added points are now marked by an A, meaning they are all Acquisition Points.
5. Press Shift $+\mathbf{N}$ (create a new file at item) on the first point and then again on the last point. In the dialog that comes up, select Montaged Images.

6. When the montage dialog pops up, set up a montage size that covers one grid square. This depends on the magnification and the mesh size of the grids used and typically requires $2 \times 2$ to $4 \times 4$ montages. Give it a name with a number (e.g., squaremap_01.mrc) to allow the SerialEM to conveniently auto-number all the files per grid square.

7. Start the grid-mapping by opening the SerialEM Navigator menu and click on Acquire At Items.

8. In the pop-up menu, select the following options.

1. Select Rough Eucentricity.

2. Select Acquire Map Image.

3. Select Close Column Valves at End.

4. Select Send Email at End.

\section{Selecting the targets}

1. Open a dummy SerialEM instance. This can be set up on the SerialEM PC that controls the microscope or on a different (support) PC if both computers share a network connection.

2. Once the first grid square is mapped, use the SerialEM Navigator menu option Merge to see the montage in the dummy SerialEM instance.

3. Double click on the Navigator window to open grid square map.

4. Search the map and use the dummy SerialEM Navigator option Add Points to add image acquisition points on the target of interest. 
5. When done and after mapping the new squares, save the Navigator file and merge the Navigator again; continue until all grid squares are mapped.

\section{Generate virtual maps}

1. Once again, merge the navigator file with the dummy SerialEM instance.

2. Run PyEM Virtual maps script from dummy SerialEM menu, Tools and select Virtual Anchor Maps. This may take some time depending on the size and amount of the grid square maps and the binning of the View and Preview maps.

NOTE: PyEM starts a command window that automatically closes when done, then the new navigator file can be opened. Per montaged map that contains selected points, PyEM writes a single merged map and all its View and Preview maps. Finally, it writes a new Navigator file called <navigatorfilename>_automaps.nav.

\section{Microscope tuning}

1. Check the microscope tuning. To assure proper microscope performance, use the same magnification and beam size setup for data acquisition, in the following order.

1. Run SeriaLEM Coma-free alignment by CTF (Zemlin tableau).

2. Insert and center an objective aperture.

3. Run SerialEM Correct Astigmatism by CTF (autostigmate).

4. Run GIF Quick Tune (i.e., only slit focus).

NOTE: As steps 7.1.1, 7.1.3, and 7.1.4 might require more dose rate, only the spot size should be changed; the beam size should not be changed as this causes beam tilt, which makes the tunings incorrect. Steps 7.1.1, 7.1.2, and 7.1.3 are semiautomated in this publicly available script (https:// serialemscripts.nexperion.net/script/47).

\section{Set up Navigator}

1. In SerialEM, open the new navigator file named <navigatorfilename>_automaps.nav.

NOTE: V_yyyy are the View maps and P_yyyy are the Preview maps. The preview maps are set as Acquisition Points.

2. In the SerialEM Navigator window, deselect all A points, select the first $\mathbf{V}$ _yyyy map, select Collapse, click on $\mathbf{A}$ twice, and deselect Collapse.

3. Select the first $\mathbf{V}$ _yyyy position, press Shift $+\mathbf{T}$, then select the very last $\mathbf{V} \_$yyyy position, and press Shift + T again.

4. Choose Single Frame Images in the properties of the file to open dialog.

5. In the next File Properties dialog, select the desired parameters according to the imaging needs and the instrument setup.

6. When prompted, give a name with a number (e.g., TS_001.mrc) and click on Save.

NOTE: SerialEM will auto-number the filenames for all tilt series.

7. Set up the tilt series controller for the first TS position. When done, click on OK to set these parameters for all tilt series after this acquisition item. All Preview maps are now selected as TS (Tilt Series) with numbered file name TS_xxx.mrc. 
NOTE: One can change the parameters manually later using the Navigator TSparams feature; changes will be applied for all items downwards on the list. The user has the option to run a custom script instead of the tilt series.

\section{Set the focus/track positions}

1. Set the focus/track distance for each target if needed (make sure that SerialEM Low Dose is switched on).

2. Double click on the View Map to load it.

3. Select the Preview Map in the Navigator list.

4. Select Edit Focus in the Navigator window.

5. In the Low Dose Control panel, deselect Rotate interarea axis to position Trial and Focus along the stage tilt axis.

6. Click on the desired region in the loaded view map to set the focus/trial position for this tilt series.

7. Ensure that the Navigator item has TSP set now; repeat the procedure for all the items.

NOTE: Focus/track positions are automatically copied downwards in the Navigator. Therefore, if the focus/track position for the previous itemis on the correct side and of the correct distance, there is no need to change it for the current item.

\section{Set up additional scripts}

1. Two scripts handle the Focus range: pretomo and duringtomo. The pretomo script runs before each tilt series and the duringtomo script during each tilt.

2. Edit the focus range in the script pretomo.

3. In the SerialEM Tilt Series menu, check Run Script in TS and select the script number of the duringtomo script.

\section{Run}

1. Check the nitrogen tank level.

2. Check whether the Autoloader turbo off is selected.

3. Check the data storage free space.

4. In the SerialEM file menu, deselect continuous saving for the log file and close any currently open logfile. Each tilt series will get its own log file.

5. In the Navigator menu, click on Acquire at Items.

6. Run the script PreTomo.

7. Select Primary task Acquire tilt series.

8. Select Run script after PostTomo.

9. Select Close column valves at end.

10. Select Send email at end.

11. Click on $\mathbf{G O}$.

NOTE: SerialEM will send an email per tilt series, either successful or error; error can, however, also mean the full tilt range was not reached.

\section{Representative Results}

This procedure was used to acquire the Sars-Cov-2 tilt series described in Turonova et al. $2020^{20}$; the whole dataset was produced using three distinct grids over three microscopy sessions at EMBL Heidelberg. The current study will focus on and describe the first 3-day $(\sim 72 \mathrm{~h})$ session run with the first grid.

After the entire grid was mapped at low magnification ( 10 min, see step 2), 71 suitable squares were selected on the grid map, and medium magnification maps (square maps) were acquired with settings (magnification, exposure, defocus) that allow for the direct visualization and 
identification of the sample of interest, coronaviruses in this case (see step 4) (Figure 1A). The acquisition time was $\sim 3$ min per square, $3 \mathrm{~h} 45 \mathrm{~min}$ in total.

As soon as the first square map was created, a dummy SerialEM instance (without any control on camera or microscope) was opened on a separate computer to visualize the square map and to add points on targets suitable for tilt series acquisition (see step 5) (Figure 1B). Newly acquired square maps were retrieved by merging the current dummy SerialEM navigator with the navigator from the acquiring SerialEM instance. After $\sim 2 \mathrm{~h}$ of grid square acquisition and selection, 50 initial targets could be identified.

After the square map acquisitions had finished, SerialEM lowdose was set up and reference View and Preview images were taken and saved as maps (see step 3). The latter maps could then be used immediately on the dummy SerialEM instance to generate, from the corresponding square map images, the Virtual View (Figure 1C) and Virtual Preview (Figure 1D) maps of the 50 selected targets with the PyEM software suite, for a processing time of $\sim 30$ min (see step 6). This processing time on the dummy SerialEM session was used to perform final preparations of the microscope for acquisition: energy filter tuning, new camera gain reference image acquisition, astigmatism- and coma-free alignment of the objective lens.
Once microscope tuning was completed and virtual maps from the 50 initial targets generated, the actual SerialEM navigator to be used for acquisition was set up (see step 8), focus and track positions were set (step 9), and tilt series acquisition could be started (see steps 10 and 11). The Virtual View maps (Figure 1C) were used for an initial centering of the target (Figure 1E) followed by a final centering performed at the actual tilt series acquisition magnification (Figure 1F) using the Virtual Preview map (Figure 1D).

Starting with grid mapping at 9:30 in the morning, the acquisition of the tilt series for the 50 initial targets commenced at roughly 15:00. With the settings used for tomographic acquisition (see the reference for details), a tilt series took $\sim 20$ min to acquire, with enough targets then to run through the entire night. While the acquisition was running, the rest of the square maps could be inspected and more targets added, still off-line via the dummy SerialEM instance. 121 more targets were selected among the remaining square maps and added to the acquisition SerialEM navigator after virtual maps had been created for these new targets, enough to run until completion of the $72 \mathrm{~h}$ session.

This procedure (summarized in Figure 2) allowed, in a single working day, the setup of 171 targets for automated tomographic acquisitions for a 72 h (3 days) microscope session. 

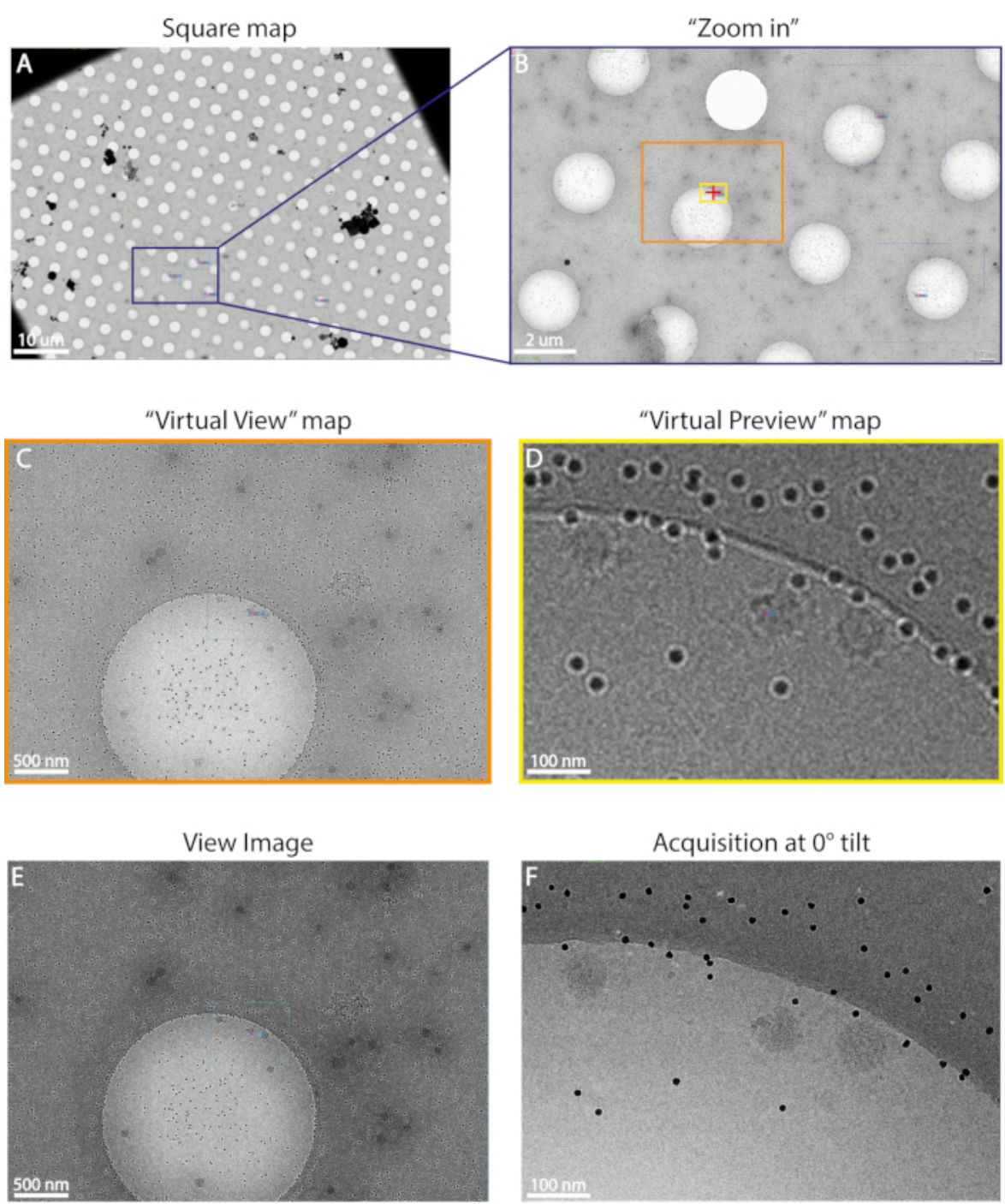

Figure 1: Example of square map with representative virtual maps and corresponding acquisitions after centering. (A) Representative square map of a Sars-Cov-2 cryoEM grid used in Turonova et al. ${ }^{20}$. Four regions of interest are marked with a red cross. Microscope magnification is 2,250x. (B) Crop out of the square map highlighting the areas that were used to generate the Virtual View (orange) and Virtual Preview (yellow) maps for the selected target (red cross) (C) Virtual View map. (D) Virtual Preview map. (E) Actual View acquisition after centering using the Virtual View map as a reference. Microscope magnification is $11,500 x$. (F) $0^{\circ}$ tilt acquisition from the tilt series after centering using the Virtual Preview map as a reference. Microscope magnification is $64,000 x$. Please click here to view a larger version of this figure. 

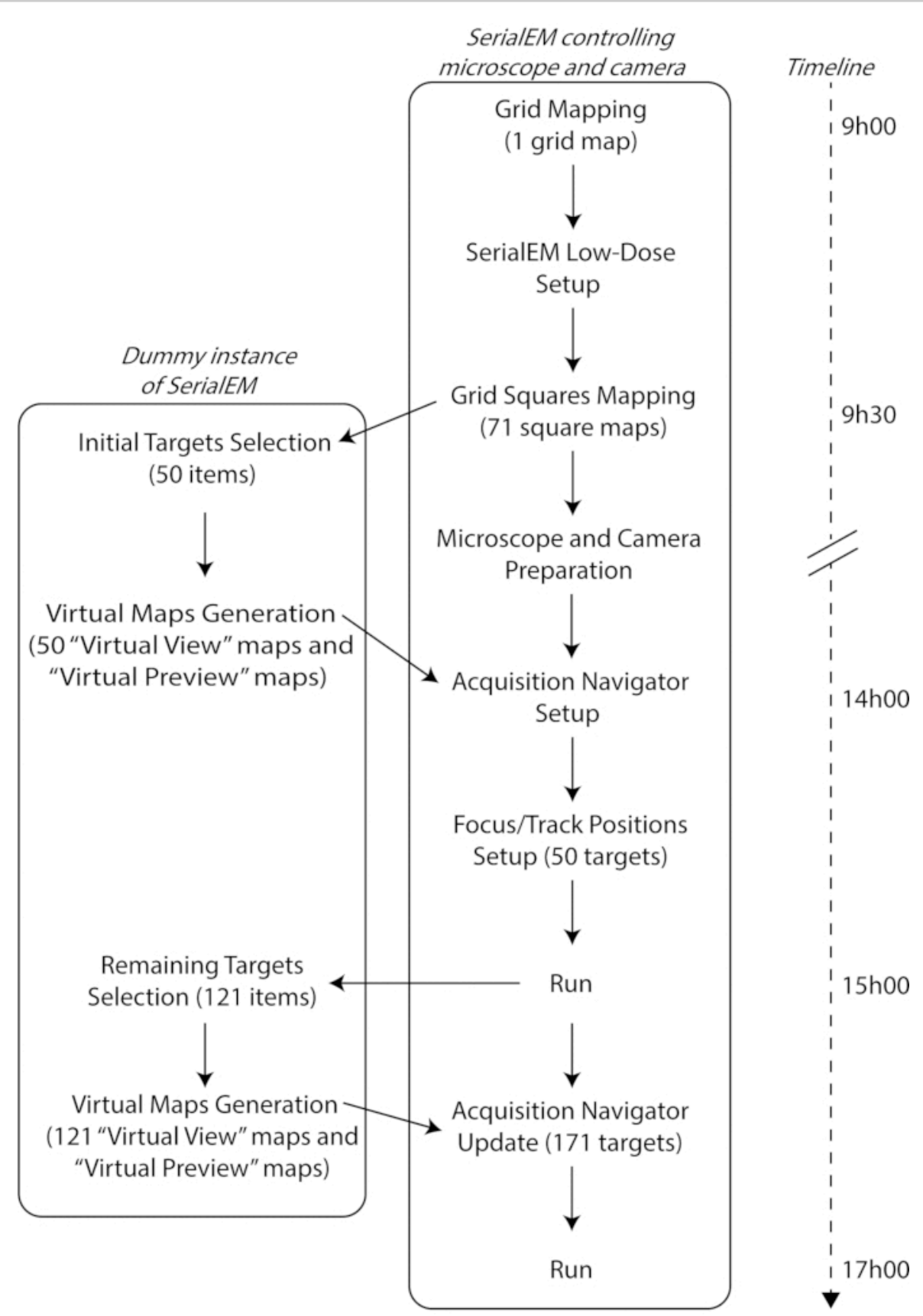

Figure 2: CryoET session workflow using SerialEM with PyEM tools. Please click here to view a larger version of this figure.

\section{Discussion}

From a niche technique, cryoET has now matured into a widespread method to perform structural studies at the cellular and molecular level with unprecedented reachable resolution $^{21,22}$. The ever-increasing demand for cryoEM imaging has put a strain on the limited resources available to access this technology. Despite the opening of a number of national cryoEM facilities and the efforts of scientific institutes 
to increase their TEM capacity to support the needs of the community worldwide, access to cryoEM instruments is still limited and the time available for data collection must therefore be efficiently used by the users to maximize the yield of each microscopy session. The need to acquire hundreds of tilt series combined with the limited time available for data collection called for novel image acquisition routines to achieve better throughput without compromising data quality. Recent developments in hardware and imaging workflows have considerably increased the speed of tilt series acquisition $^{18,19}$, thus resulting in a dramatic shift of the ratio between time spent to set up an acquisition point and time needed for the actual tilt series acquisition. Altogether, the procedure for setting up acquisition points is becoming one of the major bottlenecks for the achievable throughput of cryoET sessions.

The optimized protocol presented here enabled us to set up, in off-line mode, 171 positions for automated tomographic acquisition within the first day of a cryoET session while the microscope was actively engaged in other operations (e.g., square mapping, tuning, and automated tilt series acquisition), thus without affecting the microscope time available for data collection. In addition to maximizing the throughput of a cryoET session, this pipeline drastically reduces the amount of time invested by the user in the preparatory phase of an automated data collection session. In the described protocol, the user is asked to browse the mapped grid squares to identify suitable regions of interest and add them to the Serial EM Navigator as acquisition points. All targets will then be automatically processed in batch within SerialEM by the PyEM tool for the production of the virtual maps ${ }^{16}$. The presented computational approach is therefore considerably faster than acquiring real anchoring maps by eliminating the waiting periods associated with stage movement, image acquisition, change of imaging conditions between View and Preview, and by the eventual reiteration of these steps while centering at high magnification. Additionally, as each acquired image leads to accumulation of electron dose on the object of interest $^{23}$, the use of virtual maps for the precise realignment of the targets reduces the radiation damage introduced in the preparatory phase of a cryoET session before the actual tilt series acquisition. The protocol described here makes use of both intermediate and high magnification virtual maps (Preview and View, respectively) for realignment of the target before tilt series acquisition. This procedure can easily be modified to only use the intermediate magnification View image when alignment accuracy is of less importance, for instance, in the case of large structures where ultimate target accuracy is of less concern ${ }^{10}$ or for single particle analysis samples that are poorly spread on the cryo-grid requiring the user's manual selection of each acquisition point ${ }^{24,25}$. Finally, an approach based on the off-line use of a dummy SerialEM instance also facilitates the setup of acquisition points through remote connection by minimizing the need of the physical presence of the user at the microscope, thus enabling more flexibility in terms of operational organization of the facility.

The recent advances in technology and methods for cryoET have drastically improved the speed and reliability of automated data collection sessions. However, further developments are required to address the remaining ratelimiting steps of this method. Most notably, the initial step of grid and square mapping is now becoming one of the major bottlenecks of the session setup, thus generating the need for hardware improvements aimed at increasing the speed of microscope stage movements and of image acquisition by the direct electron detectors. Additionally, the development of 
machine learning approaches to fully automate the process of target identification will be crucial to eliminate the need of the users' visual inspection for selecting the regions of interest, a time-consuming procedure that relies on the users' expertise.

\section{Disclosures}

The authors declare no conflicts of interest.

\section{Acknowledgments}

We acknowledge support from the Structural and Computational Biology Unit and the Electron Microscopy Core Facility at the European Molecular Biology Laboratory in Heidelberg, Germany and from iNEXT-Discovery (project number 871037$)$. We are extremely grateful for the excellent support from the author of the SerialEM software package, Professor David Mastronarde. We also thank Herman Fung for the critical reading of the manuscript.

\section{References}

1. Adrian, M., Dubochet, J., Lepault, J., McDowall, A. W. Cryo-electron microscopy of viruses. Nature. 308 (5954), 32-36 (1984).

2. Cheng, Y. Single-particle cryo-EM-How did it get here and where will it go. Science. 361 (6405), 876-880 (2018).

3. Lucic, V., Forster, F., Baumeister, W. Structural studies by electron tomography: From Cells to Molecules. Annual Review of Biochemistry. 74 (1), 833-865 (2005).

4. Pfeffer, S., Mahamid, J. Unravelling molecular complexity in structural cell biology. Current Opinion in Structural Biology. 52, 111-118 (2018).

5. Schur, F. K. M. Toward high-resolution in situ structural biology with cryo-electron tomography and subtomogram averaging. Current Opinion in Structural Biology. 58, 1-9 (2019).

6. Bohning, J., Bharat, T. A. M. Towards high-throughput in situ structural biology using electron cryotomography. Progress in Biophysics and Molecular Biology. In Press (2020).

7. Briggs, J. A. Structural biology in situ--the potential of subtomogram averaging. Current Opinion in Structural Biology. 23 (2), 261-267 (2013).

8. Wan, W., Briggs, J. A. G. Methods in Enzymology.Elsevier, NY. 579, 329-367 (2016).

9. Tan, Y. Z., Cheng, A., Potter, C. S., Carragher, B. Automated data collection in single particle electron microscopy. Microscopy. 65 (1), 43-56 (2016).

10. Morado, D. R., Hu, B., Liu, J. Using Tomoauto: A protocol for high-throughput automated cryo-electron tomography. Journal of Visualized Experiments: JoVE. 107, e53608 (2016).

11. Resch, G. P. in Methods in Cell Biology. Elseiver, NY. 152, 135-178 (2019).

12. Bharat, T. A., Kukulski, W. Correlative Imaging: Focusing on the Future. John Wiley \& Sons, NY. 137-153 (2019).

13. Hagen, W. J. H., Wan, W., Briggs, J. A. G. Implementation of a cryo-electron tomography tiltscheme optimized for high resolution subtomogram averaging. Journal of Structural Biology. 197 (2), 191-198 (2017).

14. O'Toole, E., van der Heide, P., Richard McIntosh, J., Mastronarde, D. Cellular Imaging: Electron Tomography and Related Techniques. Springer, Cham.95.-116 (2018). 
15. Mastronarde, D. N. Automated electron microscope tomography using robust prediction of specimen movements. Journal of Structural Biology. 152 (1), 36-51 (2005).

16. Schorb, M., Haberbosch, I., Hagen, W. J. H., Schwab, Y., Mastronarde, D. N. Software tools for automated transmission electron microscopy. Nature Methods. 16, 471-477 (2019).

17. Turonova, B. et al. Benchmarking tomographic acquisition schemes for high-resolution structural biology. Nature Communications. 11 (1), 876 (2020).

18. Chreifi, G., Chen, S., Metskas, L. A., Kaplan, M., Jensen, G. J. Rapid tilt-series acquisition for electron cryotomography. Journal of Structural Biology. 205. (2), 163-169 (2019).

19. Eisenstein, F., Danev, R., Pilhofer, M. Improved applicability and robustness of fast cryo-electron tomography data acquisition. Journal of Structural Biology. 208 (2), 107-114 (2019).

20. Turonova, B. et al. In situ structural analysis of SARSCoV-2 spike reveals flexibility mediated by three hinges. Science. 370 (6513), 203-208 (2020).

21. O'Reilly, F. J. et al. In-cell architecture of an actively transcribing-translating expressome. Science. 369 (6503), 554-557 (2020).

22. Tegunov, D., Xue, L., Dienemann, C., Cramer, P., Mahamid, J. Multi-particle cryo-EM refinement with $M$ visualizes ribosome-antibiotic complex at $3.7 \AA$ inside cells. Nature Methods. 18., 186-193 (2020).

23. Karuppasamy, M., Karimi Nejadasl, F., Vulovic, M., Koster, A. J., Ravelli, R. B. G. Radiation damage in single-particle cryo-electron microscopy: effects of dose and dose rate. Journal of Synchrotron Radiation. 18 (3), 398-412 (2011).

24. Liberta, F. et al. Cryo-EM fibril structures from systemic AA amyloidosis reveal the species complementarity of pathological amyloids. Nature Communications. 10 (1), 1104 (2019).

25. Radamaker, L. et al. Cryo-EM structure of a light chain-derived amyloid fibril from a patient with systemic AL amyloidosis. Nature Communications. 10 (1), 1103 (2019). 